\title{
Topographically Selective Reinnervation of Adult Mammalian Skeletal Muscles
}

\author{
Michael B. Laskowski and Joshua R. Sanes \\ Department of Anatomy and Neurobiology, Washington University School of Medicine, St. Louis, Missouri 63110
}

In 2 rat muscles, serratus anterior and the diaphragm, the rostrocaudal axis of the motor pool is mapped onto the rostrocaudal axis of the muscle's surface (Laskowski and Sanes, 1987a). One possible basis for this orderly topography is that motor axons and intramuscular structures bear labels that favor connectivity among positionally matched partners. To test for the existence of such labels, we asked whether axons would selectively reinnervate appropriate portions of the muscles following nerve transection. We found that, on average, rostral and caudal halves of each muscle were preferentially reinnervated by axons from the rostral and caudal halves of its motor pool, respectively. In the serratus anterior, reinnervation was more selective following denervation in neonates than following denervation in adults, although in neither case was the normal pattern of innervation reestablished completely. These results show that motor axons can selectively reinnervate adult rat muscles, and support the idea that positional cues play a role in organizing neuromuscular topography.

Topographically organized patterns of connectivity are a common feature of both the central and peripheral nervous systems. Within the motor system, muscles that arise from segmentally arranged myotomes are generally innervated by motoneurons from corresponding levels of the neuraxis (reviewed in Fetchko, 1987). We recently showed that connectivity is organized in the rostrocaudal axis even within individual axial muscles: in both the anterior serratus and the diaphragm, the rostrocaudal axis of the motor pool maps systematically onto the rostrocaudal axis of the muscle surface (Laskowski and Sanes, 1987a). Limb muscles also receive topographically ordered projections from their motor pools (Swett et al. 1970; Brown and Booth, 1983; Bennett and Lavidis, 1984). One possible explanation for the gencsis of these maps is that motor axons and some intramuscular structures bear labels that favor synapse formation between positionally matched synaptic partners. However, the adult pattern alone does not provide compelling evidence on this point because topographically organized connectivity could arise from spatiotemporally coordinated patterns of axon elongation and muscle growth in the embryo. More direct evidence would

\footnotetext{
Received Sept. 15, 1987; accepted Dec. 2, 1987.

We thank J. Cunningham and S. Eadis for assistance, V. Friedman for illustrations, and D. Hunter, D. Purves, L. Scott, and D. Wigston for comments. This work was supported by the Muscular Dystrophy Association and the National Institutes of Health.

Correspondence should be addressed to Michael B. Laskowski at his permanent address: WAMI Regional Medical Program, University of Idaho, Moscow, ID 83843 .

Copyright (C) 1988 Society for Neuroscience $0270-6474 / 88 / 083094-06 \$ 02.00 / 0$
}

be the demonstration that motor axons selectively reinnervate adult muscles in a topographically appropriate manner. In several previous studies of adult mammalian muscles, reinnervation was reported to be nonselective (Weiss and Hoag, 1946; Bernstein and Guth, 1961; Miledi and Stefani, 1969; Gerding et al., 1977; Riley, 1978; Brushart and Mesulam, 1980; Mizuno et al., 1980; Gillespie et al., 1986; see also Aldskogius and Thomander, 1986; Hardman and Brown, 1987). However, these studies did not systematically evaluate rostrocaudal position. Moreover, we previously showed that sympathetic preganglionic axons can selectively reinnervate adult rat intercostal muscles in a way that reflects their position in the rostrocaudal axis (Wigston and Sanes, 1982, 1985). On the basis of this result, we were encouraged to assess the reinnervation of rostral and caudal portions of the diaphragm and serratus anterior by rostral and caudal portions of their respective motor pools. We found that reinnervation is positionally selective in both muscles, although the graded topographic map is not completely restored in either case. These results demonstrate that adult mammalian motor axons can selectively reinnervate skeletal muscles to some degree, and provide evidence for the existence of positional cues that modulate synaptic connectivity within a single muscle.

An abstract of this work has appeared previously (Laskowski and Sanes, 1987b).

\section{Materials and Methods}

The 2 muscles used, the diaphragm and serratus anterior, are sketched in Figure 1. Each muscle is innervated by a single nerve that carries motor axons from more than one spinal cord segment. The muscles are thin and flat, and have clear subdivisions (sectors) that form a rostrocaudal progression. For surgery, female Sprague-Dawley rats 4-5 weeks old $(60-90 \mathrm{gm})$ were anesthetized with chloral hydrate. By this age, synapse elimination is complete in all rat muscles that have been studied (Grinnell and Herrera, 1981), including the diaphragm ( $\sim 14 \mathrm{~d}$; Rosenthal and Taraskevich, 1977), and animals are approaching sexual maturity (weaning, $\sim 21 \mathrm{~d}$; first estruis, $\sim 35 \mathrm{~d}$; puberty, $\sim 50 \mathrm{~d}$; Baker et al., 1979). To denervate the serratus anterior, an incision was made above the right axillary space, and the descending thoracic nerve was transected as it entered the muscle. For the diaphragm, an incision was made in the right thorax and the phrenic nerve transected as it coursed along the inferior vena cava approximately $1-2 \mathrm{~mm}$ before it entered the diaphragm. In one set of experiments, the nerve to the serratus anterior was cut in a group of neonates on postnatal day 1 . In all cases, it was important that the nerve be transected completely to avoid the possibility that axons could be passively guided along preexisting pathways to their original targets. Thus, when the nerve was cut, we confirmed that there was a gap between its proximal and distal ends and that each end could be lifted away from the underlying tissue (Fig. 2A). During the course of reinnervation, neurites grew beyond the perineural sheath of the proximal stump to reach the distal stump (Fig. 2, $B, C$ ).

Reinnervation was assessed 4-11 weeks after the nerve was cut, using methods described fully in Laskowski and Sanes (1987a). Briefly, ani- 

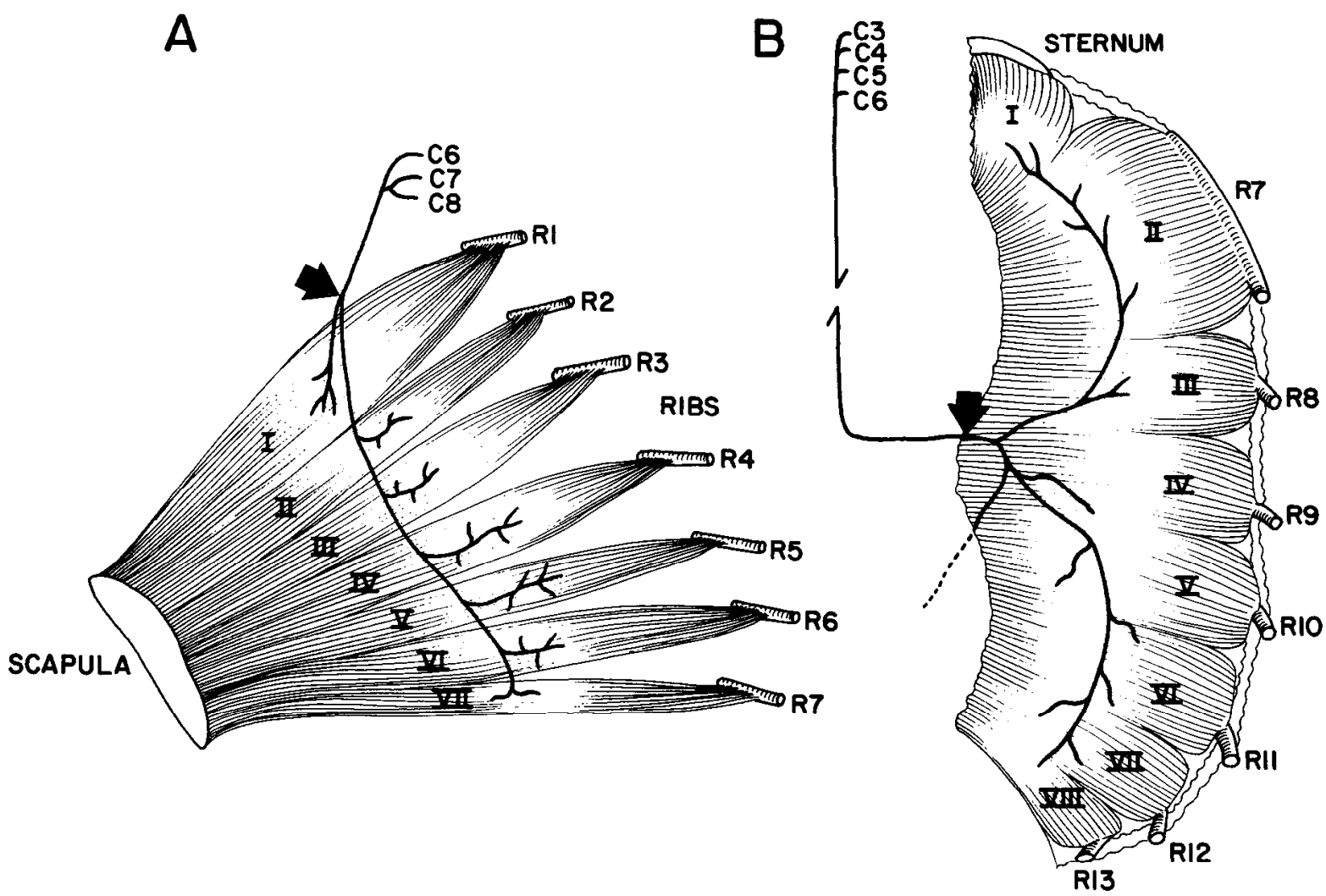

Figure 1. Drawings of muscles used in this study. $A$, The anterior serratus consists of 7 sectors formed by the origin of muscle fibers from ribs 1-7. The long thoracic nerve, which arises from $\mathrm{C} 6-\mathrm{C} 8$, innervates the serratus. $B$, The hemidiaphragm consists of 8 sectors formed by the origin of its fibers from connective tissue associated with the sternum and with ribs 7-13. Innervation is provided by the phrenic nerve, which arises from ventral roots $\mathrm{C} 3-\mathrm{C} 6$. Arrows mark sites at which nerves were transected. Drawings are not to scale.

mals were anesthetized with chloral hydrate and perfused through the aorta with mammalian Ringer's solution. The muscle, its nerve, and the relevant ventral roots were then dissected in continuity, and the preparation was mounted in a recording chamber. Some ventral roots were separated into bundles of rootlets along natural subdivisions, and each root or group of rootlets was pulled into a suction electrode for stimulation. For the anterior serratus, the following cervical $(C)$ roots or groups of rootlets were stimulated: $\mathrm{C} 6$ rostral (C6R), $\mathrm{C} 6$ intermediate (C6I), C6 caudal (C6C), $\mathrm{C} 7$ and $\mathrm{C} 8$; for the diaphragm: $\mathrm{C} 3, \mathrm{C} 4$ rostral (C4R), C4 caudal (C4C), C5 rostral (C5R), C5 caudal (C5C), and C6. The organization of the muscles we used, in which individual sectors originate from different ribs, makes the identification of the sectors that contract upon stimulation of any particular root or rootlet straightforward. Localized contractions in each muscle sector were rated on a scale of 0 (no visible contraction) to 4 (strongest contraction observed). The muscle was then stretched to reduce movement, and muscle fibers were impaled in the endplate zone with a $\mathrm{KCl}$-filled microelectrode. During each impalement, all ventral roots or rootlets were stimulated in turn, and synaptic potentials or action potentials recorded to determine the source of the reinnervating axons. Few responses were recorded from $\mathrm{C} 3$ in the diaphragm, and none from $\mathrm{C} 8$ in the serratus anterior; these roots are therefore not shown in Figures 3-5. In general, recordings were obtained from 10 muscle fibers in each sector of each muscle. Data from control muscles have been replotted from Laskowski and Sanes (1987a) to permit comparison with new results on reinnervated muscles.

\section{Results}

\section{Reinnervation of the serratus anterior}

In adult rats, the rostrocaudal axis of the serratus anterior motor pool is mapped systematically onto the rostrocaudal axis of the serratus anterior muscle. This mapping is not point-to-point, with nearly all portions of the motor pool contributing some input to nearly all portions of the muscle. Rather, progressively more caudal sectors of the muscle receive the bulk of their innervation from progressively more caudal regions of its motor pool (Laskowski and Sanes, 1987a). Our aim here was to learn whether rostral and caudal portions of the muscle are selectively reinnervated by rostral and caudal portions of the motor pool following nerve transection in the adult.

By 3-4 weeks after denervation, considerable regeneration had occurred: the entire muscle contracted when the nerve was stimulated, and synaptic responses were recorded intracellularly from muscle fibers in all 7 sectors. It was immediately apparent that the motor pool-to-muscle map was not faithfully restored upon reinnervation, and, in the limited number of muscles examined, an orderly rostrocaudal gradient of connectivity was not detectable. Nonetheless, comparison of the rostral and caudal halves of the muscle and of the motor pool revealed that reinnervation was indeed positionally selective. We documented this selectivity in 2 different ways.

First, we constructed an index reflecting the average segmental origin of all inputs to each half of the anterior serratus. As detailed previously (Wigston and Sanes, 1982, 1985; Laskowski and Sanes, 1987a), roots or rootlets were numbered in order $(\mathrm{C} 6 \mathrm{R}=1, \mathrm{C} 6 \mathrm{I}=2, \mathrm{C} 6 \mathrm{C}=3, \mathrm{C} 7=4)$, and these numbers were used to calculate the average source of inputs to each portion of the muscle. For example, we recorded 4 inputs from C6R, 1 from $\mathrm{C} 6 \mathrm{I}, 9$ from $\mathrm{C} 6 \mathrm{C}$, and 13 from $\mathrm{C} 7$ in the caudal half(sectors V-VII) of one reinnervated serratus. This half-muscle received a score of $4(1)+1(2)+9(3)+13(4) /(4+1+9+13)=3.15$. Thus, by this measure, muscles innervated by more caudally 

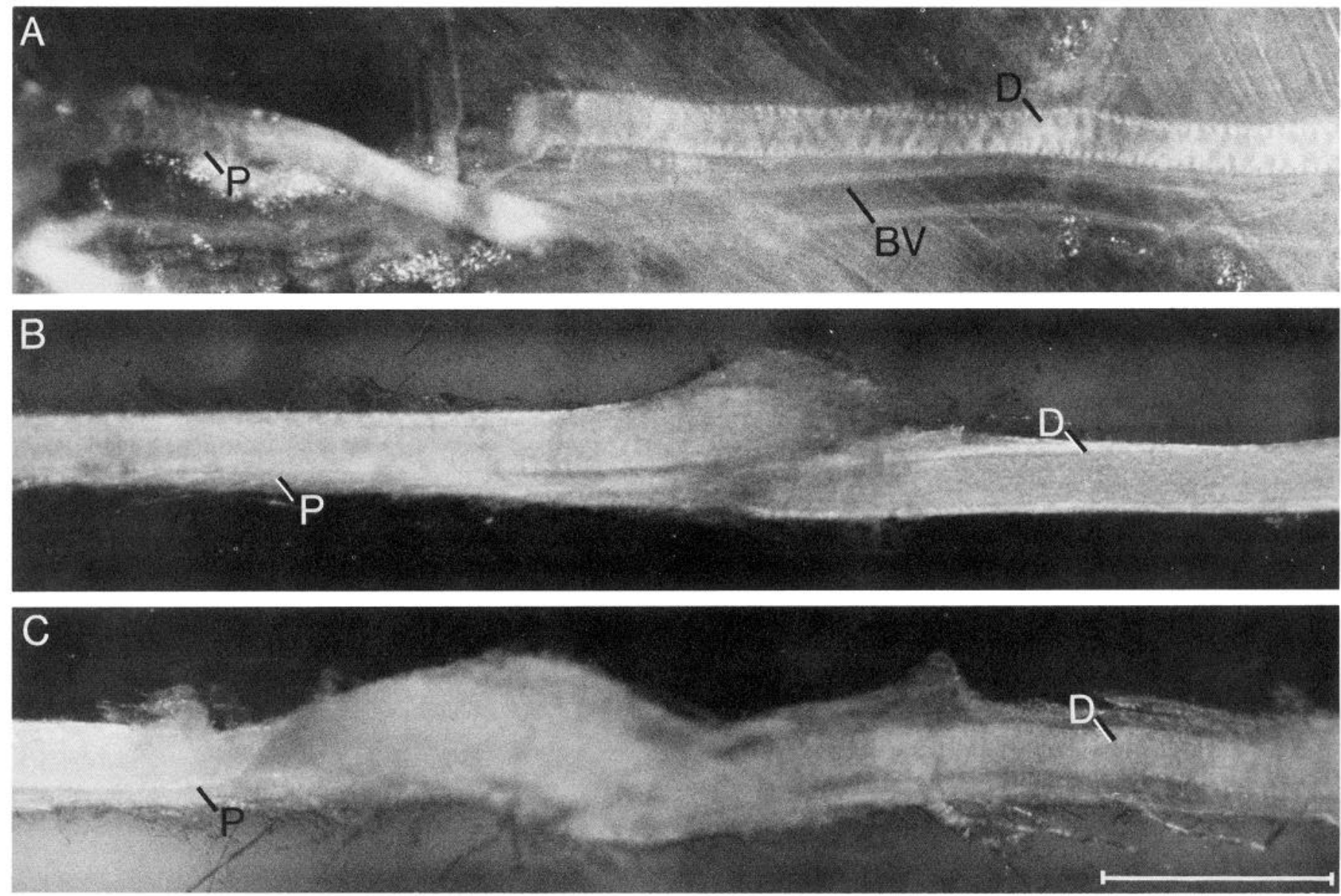

Figure 2. The long thoracic nerve $1(A), 11(B)$, or $23(C) \mathrm{d}$ after transection. Nerves in $B$ and $C$ were freed of connective tissue and muscle for photography, but relationships of proximal $(P)$ to distal $(D)$ stumps were not disturbed. The nerve was completely transected, so that neurites were forced to grow outside of endoneurial and perineural guides before reinnervating muscle. Bar, $1 \mathrm{~mm}$.

derived axons receive higher values of segmental innervation. A similar index was constructed to summarize localized contractile responses (rated $0-4$ ) to stimulation of individual roots or rootlets. Using either intracellularly recorded responses or localized contractions as measures of reinnervation, these indices demonstrated that rostrally and caudally derived subsets of axons preferentially reinnervated rostral and caudal halves, respectively, of the serratus anterior (Fig. 3, top).

A second way of analyzing the same data was to construct an analogous index that reflected the center of the area that each ventral root innervated within the serratus muscle. For example, in response to stimulation of one $\mathrm{C} 7$ root, we recorded synaptic potentials from one muscle fiber in sector II, 2 in sector V, 8 in sector VI, and 3 in sector VII. In this muscle, the C7 root was scored as having an average target field of $1(2)+2(5)+$ $8(6)+3(7) /(1+2+8+3)=5.78$. Such indices, calculated using either intracellular responses or localized contractions, showed that axons in the $\mathrm{C} 6$ ventral root reinnervated a more rostral subset of muscle fibers within the serratus than did axons in the $\mathrm{C} 7$ root (Fig. 3, bottom).

Three aspects of the reinnervation that are relevant to our tabulation of results need to be noted. First, intracellular responses included both action potentials and subthreshold potentials. The latter were generally recorded when the muscle fiber's resting potential was low $(<-65 \mathrm{mV})$, and probably rep- resented aborted action potentials rather than true, nonpropagating postsynaptic potentials. Both were considered together in tabulating intracellular responses. Second, in muscles studied $26-35 \mathrm{~d}$ after denervation, about $5 \%$ of the muscle fibers sampled (16 of 340) received inputs from 2 different roots or rootlets. Such polyaxonal innervation was rare in muscles studied at later times ( 0 of 200 fibers) or in control muscles ( 1 of 680 fibers). A transient stage of polyaxonal reinnervation has previously been noted in other studies of mammalian muscle (McArdle, 1975; Gorio et al., 1983). Our results were not significantly affected by inclusion of polyaxonally innervated fibers; results from all fibers were therefore combined in Figure 3. Finally, and most important, during the course of our experiments we discovered that we had failed to cut the nerve branch supplying sector $I$ in a few muscles, thereby leaving that sector innervated (see Fig. 1A). We have therefore discarded all responses from this sector in tabulating our results. Although this omission is prudent, it results in a significant underrepresentation of the selectivity that was actually observed in both normal and reinnervated muscles.

\section{Reinnervation of diaphragm}

In the diaphragm, as in the serratus anterior, the rostrocaudal axis of the motor pool is mapped onto the rostrocaudal axis of the muscle (Laskowski and Sanes, 1987a). In a series of animals, 


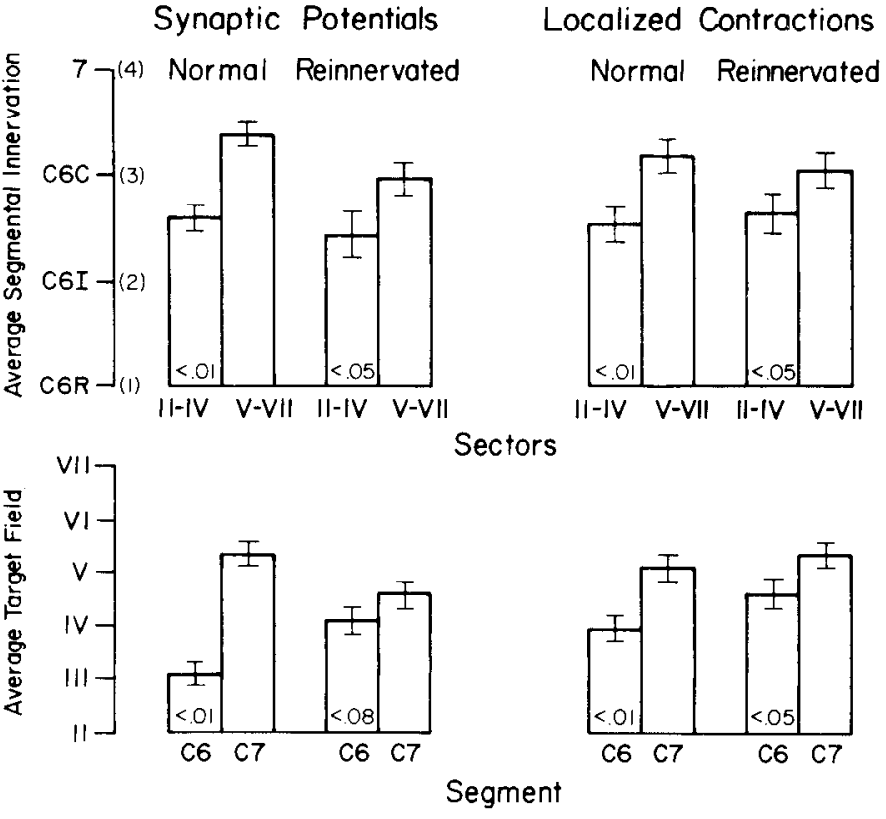

Figure 3. Topographically selective innervation and reinnervation of the anterior serratus muscle. The average segmental innervation of each half of the muscle and the average target field of each ventral root were calculated as described in Results, on the basis of synaptic potentials recorded intracellularly and localized contractions rated visually. Values are means \pm SE for 10 control and 12 reinnervated muscles. Numbers within bars indicate the significance of differences between rostral and caudal muscle sectors or nerve roots, calculated using Student's $t$ test.

we transected the phrenic nerve near the edge of the diaphragm, and then assessed reinnervation, as described above, for the serratus. Again, rostral and caudal portions of the motor pool preferentially reinnervated rostral and caudal portions of the muscle, respectively, as measured by indices of average segmental innervation and target field, calculated both for intracellularly recorded responses and for localized contractions (Fig. 4). However, the degree of selectivity was small, so that while measures of average target field differed significantly for $\mathrm{C} 4$ and C5 axons, the difference in average segmental innervation between rostral and caudal halves of the muscle did not attain statistical significance.

\section{Reinnervation of neonatally denervated serratus anterior}

In 2 recent studies, selective reinnervation of mammalian skeletal muscle was observed following denervation of neonatal muscles, but not following denervation of adult muscles (Aldskogius and Thomander, 1986; Hardman and Brown, 1987). In view of these results, we assessed the reinnervation of the anterior serratus in a series of animals that had been denervated within $24 \mathrm{hr}$ of birth. As shown in Figure 5, reinnervation was more selective following neonatal denervation than following adult denervation.

\section{Discussion}

In 2 axial muscles, the serratus anterior and the diaphragm, the rostrocaudal axis of the motor pool projects systematically onto the rostrocaudal axis of the muscle (Laskowski and Sanes, 1987a). We show here that reinnervation restored this map, albeit imperfectly, following nerve section. While the selectivity was limited in extent, it was consistent: we observed it with each of 2 measures (intracellular recording and estimation of contractile

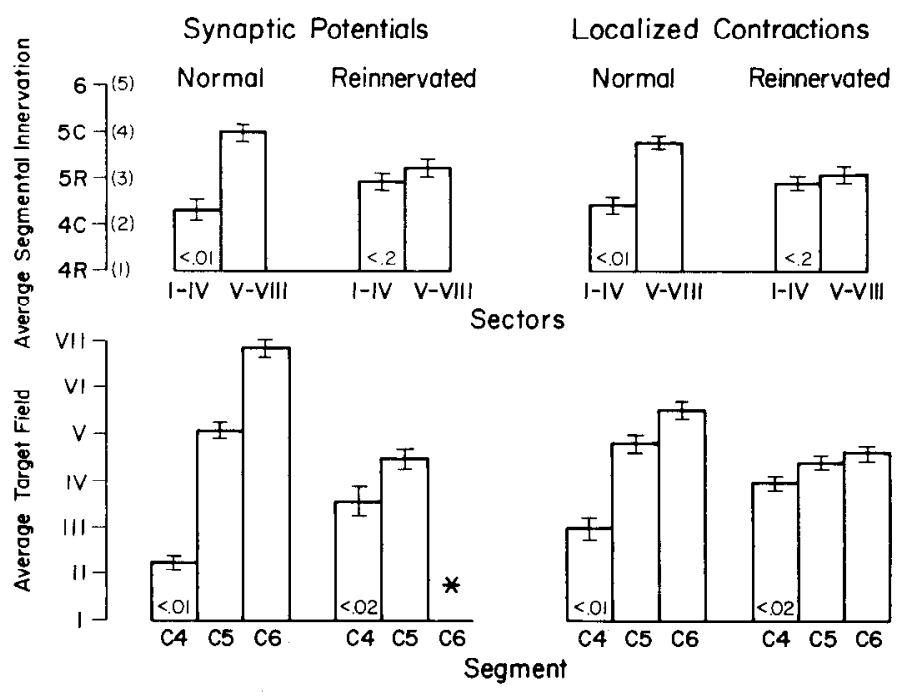

Figure 4. Topographically selective innervation and reinnervation of the diaphragm. Results are presented as in Figure 3. Localized contractions were rated in 12 normal and 14 reinnervated muscles; of these, 8 normal and 9 reinnervated muscles were studied by intracellular recording. Synaptic potentials in response to $\mathrm{C} 6$ stimulation were recorded from only 3 muscles; the average target field of this root $\left(^{*}\right)$ is therefore not shown. Iower panels, numbers with in bars indicate the significance of differences between $\mathrm{C} 4$ and $\mathrm{C} 5$ roots.

vigor), using each of 2 indices (average segmental innervation and average target field) in each of 3 series of muscles (serratus anterior following neonatal and adult denervation and diaphragm following adult denervation). These results are significant in 2 respects. First, they demonstrate that adult mammalian motor axons can selectively reinnervate appropriate muscle fibers, even within a single muscle. Second, they provide evidence for the existence of cues that bias the formation of synapses between motor neurons and muscle fibers that are positionally matched in the rostrocaudal axis.

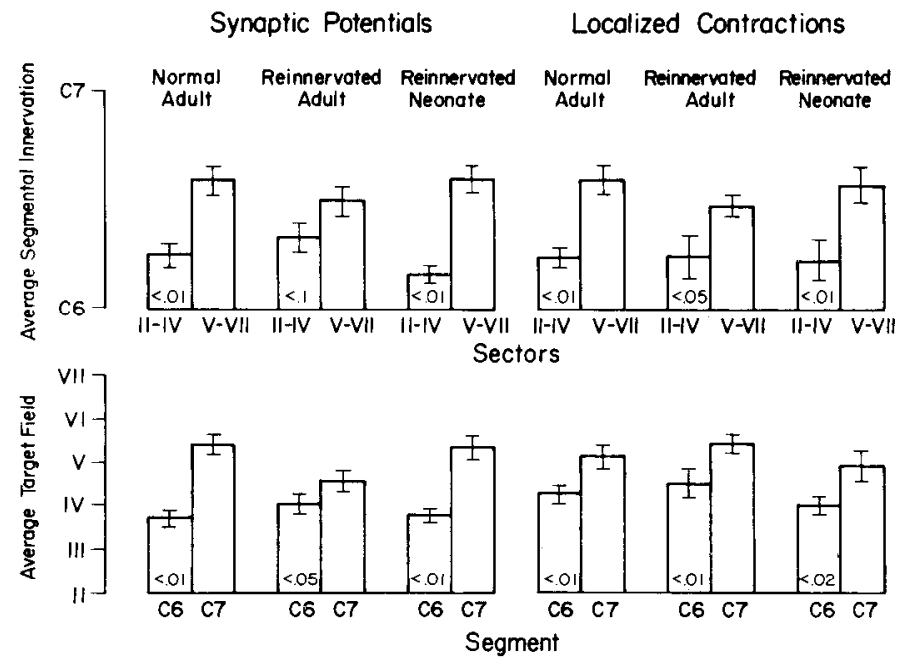

Figure 5. Topographically selective reinnervation of serratus anterior muscle following nerve transection in neonates or young adults. Results for normal and reinnervated adult muscles are from Figure 3, but medsures of average segmental innervation (and of statistical significance) are recalculated to combine all responses from $\mathrm{C} 6$. This is because the C6 root was not subdivided in the 6 animals studied following neonatal denervation. 


\section{Selective reinnervation}

The selective reinnervation reported here contrasts with previous reports of reinnervation of adult rat muscles that was found to be nonselective (Weiss and Hoag, 1946; Bernstein and Guth, 1961; Miledi and Stefani, 1969; Gerding et al., 1977; Riley, 1978; Brushart and Mesulam, 1980; Mizuno et al., 1980; Aldskogius and Thomander, 1986; Gillespie et al., 1986; Hardman and Brown, 1987). Several factors may account for the difference between our results and those of others. First, the variable we evaluated was rostrocaudal position. This choice was based on the knowledge that autonomic preganglionic axons can reinnervate both sympathetic ganglia (Purves et al., 1981) and skeletal muscles (Wigston and Sanes, 1982, 1985) with a selectivity that reflects the place of origin of the synaptic partners in the rostrocaudal axis (see below). In contrast, in most previous work, pairs of limb muscles that derive innervation from, and may themselves arise from, overlapping segmental levels werc studied (e.g., Weiss and Hoag, 1946). Second, by cutting the motor nerve near the muscle's edge and studying reinnervation within a single muscle, we maximized the opportunity for intramuscular cues to influence regenerating axons. In contrast, studies of reinnervation in adult muscle have often involved transection of a nerve proximal to an extramuscular branch point (e.g., Bernstein and Guth, 1961; Brushart and Mesulam, 1980). In these cases, nonselective axonal regeneration along extramuscular pathways might have masked intramuscular selectivity. In fact, it is our impression that the poorly selective reinnervation of the diaphragm is attributable in part to nonselective axonal growth at the extramuscular bifurcation (Fig. $1 B$ ) - a point at which axons from different segments normally take different courses; see Laskowski and Sanes (1987a)-with selectivity manifest only within each quadrant (not shown). Third, we used young adult rats $(\sim 1$ month old at denervation), whereas several previous workers used older $(\geq 200 \mathrm{gm}$ or 5-month-old) rats (e.g., Brushart and Mesulam, 1980; Gillespie et al., 1986). While our work was in progress, 2 studies appeared reporting selective reinnervation of rat muscles that were denervated neonatally; in both cases, reinnervation was nonselective following denervation in adults (Aldskogius and Thomander, 1986; Hardman and Brown, 1987; see also Gerding et al., 1977; Brown and Booth, 1983; Bennett and Lavidis, 1984). Our comparison of animals denervated neonatally and as young adults supports the notion that animals exhibit more pronounced selectivity following early denervation. Although we chose to use young adults for purely technical reasons (to minimize buildup of connective tissue after surgery, thereby facilitating subsequent recording), our choice may have serendipitously favored the detection of selective reinnervation. Finally, while the selectivity we observed was statistically significant, it was subtle and could have been overlooked in experiments designed to seek complete restoration of a normal pattern of connectivity. In short, while our work provides new evidence for selectivity in the reinnervation of adult mammalian muscles, there is no direct conflict between previous work and our results.

\section{Positional cues and selective synapse formation}

The starting point for this study was the observation that the rostrocaudal axes of the serratus anterior and phrenic motor pools are systematically mapped onto the rostrocaudal axes of the serratus anterior and diaphragm, respectively (Laskowski and Sanes, 1987a). In considering the basis for these maps, we suggestcd that motor axons and some intramuscular structures (e.g., muscle fibers, extracellular matrix, or nerve branches) might bear labels that favor synapse formation between positionally matched partners. We realized, however, that coordinated spatiotemporal patterns of axonal growth and muscle development in the embryo could also lead to the generation of a map, without the involvement of specific molecular cues. The finding that reinnervation is positionally selective when all axons regenerate together into a completely formed muscle favors the first alternative. A study of selective reinnervation of axolotl muscles has led Wigston and Kennedy (1987) to a similar conclusion. It remains unknown whether positional cues of the sort that act during reinnervation are also present in the embryo; however, they are present soon after birth and, if anything, are more potent in neonates than in adults.

The results presented here complete a set of studies of positionally selective reinnervation in the mammalian peripheral nervous system. Purves et al. (1981) showed that sympathetic ganglia transplanted from a midthoracic level to the bed of the superior cervical ganglion are reinnervated by a more caudal subset of preganglionic axons than are reimplanted superior cervical ganglia. Subsequently, Wigston and Sanes (1982, 1985) found that intercostal muscles transplanted from different segmental levels to a common site in the neck are selectively reinnervated by different subsets of preganglionic axons in accordance with their position of origin in the rostrocaudal axis. Here, we have demonstrated positional selectivity completely within the somatic motor system. Together, these results argue for the existence of a single system of positional cues shared by the sympathetic and motor systems. In all 3 cases, connectivity is not rigidly specified, in that axons from virtually any part of the spinal cord can innervate or reinnervate muscles or ganglia from any level tested. Instead, specificity is probabilistic, such that the likelihood that innervation derives from a given spinal level changes gradually from the rostral to caudal ends of the target field. This graded selectivity is consistent with the existence of gradients of molecules that bias, but do not rigidly determine, synapse formation between positionally matched partners. It will now be important to seek molecular probes that distinguish rostral from caudal motor axons or rostral from caudal muscle fibers.

\section{References}

Aldskogius, H., and L. Thomander (1986) Selective reinnervation of somatotopically appropriate muscles after facial nerve transection and regeneration in the neonatal rat. Brain Res. 375: 126-134.

Baker, H. J., J. R. Lindsey, and S. H. Weisbroth (eds.) (1979) The Laboratory Rat. Vol. 1. Biology and Diseases, Academic, New York.

Bennett, M. R., and N. A. Lavidis (1984) Development of the topographical projection of motor neurons to a rat muscle accompanies loss of polyneuronal innervation. J. Neurosci. 4: 2204-2212.

Bernstein, J. J., and L. Guth (1961) Nonselectivity in the establishment of neuromuscular connections following nerve regeneration in the rat. Exp. Neurol. 4: 262-275.

Brown, M. C., and C. M. Booth (1983) Postnatal development of the adult pattern of motor axon distribution in rat muscle. Nature 304 : 741-742.

Brushart, T. M., and M.-M. Mesulam (1980) Alteration in connections between muscle and anterior horn motoneurons after peripheral nerve repair. Science 208: 603-605.

Fetchko, J. R. (1987) A review of the organization and evolution of motoneurons innervating the axial musculature of vertebrates. Brain Res. Rev. 12: 243-280.

Gerding, R., N. Robbins, and J. Antosiak (1977) Efficiency of rein- 
nervation of neonatal rat muscle by original and foreign nerves. Dev. Biol. 61: 177-183.

Gillespie, M. J., T. Gordon, and P. R. Murphy (1986) Reinnervation of the lateral gastrocnemius and soleus muscles by their common nerve. J. Physiol. (Lond.) 372: 485-500.

Gorio, A., G. Carmignoto, M. Finesso, P. Polato, and M. G. Nunzi (1983) Muscle reinnervation. II. Sprouting, synapse formation and repression. Neuroscience $8: 403-416$.

Grinnell, A. D., and A. A. Herrera (1981) Specificity and plasticity of neuromuscular connections: Long-term regulation of motoneuron function. Prog. Neurobiol. 17: 203-282.

Hardman, V. J., and M. C. Brown (1987) Accuracy of reinnervation of rat internal intercostal muscles by their own segmental nerves. J. Neurosci. 7: 1031-1036.

Laskowski, M. B., and J. R. Sanes (1987a) Topographic mapping of motor pools onto skeletal muscles. J. Neurosci. 7: 252-260.

Laskowski, M. B., and J. R. Sanes (1987b) Topographically selective reinnervation of adult mammalian muscle. Soc. Neurosci. Abstr. 13: 1422.

McArdle, J. J. (1975) Complex and end-plate potentials at the regenerating neuromuscular junction of the rat. Exp. Neurol, 49:629-638.

Miledi, R., and E. Stefani (1969) Nonselective reinnervation of the slow and fast muscle fibers in the rat. Nature 222: 569-571.

Mizuno, N., M. Uemura-Sumi, K. Matsuda, Y. Takeuchi, M. Kume, and R. Matsushima (1980) Non-selective distribution of hypoglos- sal nerve fibers after section and resuture: A horseradish peroxidase study in the cat. Neurosci. Lett. 19: 33-37.

Purves, D., W. Thompson, and J. W. Yip (1981) Reinnervation of ganglia transplanted to the neck from different levels of the guinea pig sympathetic chain. J. Physiol. (Lond.) 313: 49-63.

Riley, D. A. (1978) An investigation of selective reinnervation of rat skeletal muscles. Soc. Neurosci. Abstr. 4: 534.

Rosenthal, J. L., and P. S. Taraskevich (1977) Reduction of multiaxonal innervation at the neuromuscular junction of the rat during development. J. Physiol. (Lond.) 270: 299-310.

Swett, J. E., E. Elred, and J. S. Buchwald (1970) Somatotopic cordto-muscle relations in efferent innervation of cat gastrocnemius. Am. J. Physiol. 219: 762-766.

Weiss, P., and A. Hoag (1946) Competitive reinnervation of rat muscles by their own and foreign nerves. J. Neurophysiol. 9: 413-418.

Wigston, D. J., and P. R. Kennedy (1987) Selective reinnervation of transplanted muscles by their original motoneurons in the axolotl. $\mathbf{J}$. Neurosci. 7: 1857-1865.

Wigston, D. J., and J. R. Sanes (1982) Selective reinnervation of adult mammalian muscles by axons from different segmental levels. Nature 299: 464-467.

Wigston, D. J., and J. R. Sanes (1985) Selective reinnervation of intercostal muscles transplanted from different segmental levels to a common site. J. Neurosci. 5: 1208-1221. 